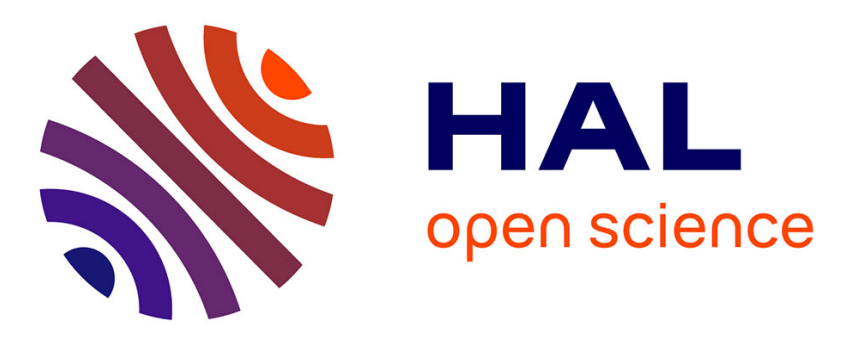

\title{
Quantum confinement and dielectric profiles of colloidal nanoplatelets of halide inorganic and hybrid organic-inorganic perovskites
}

Daniel Sapori, Mikael Kepenekian, Laurent Pedesseau, Claudine Katan, Jacky Even

\section{To cite this version:}

Daniel Sapori, Mikael Kepenekian, Laurent Pedesseau, Claudine Katan, Jacky Even. Quantum confinement and dielectric profiles of colloidal nanoplatelets of halide inorganic and hybrid organicinorganic perovskites. Nanoscale, 2016, Themed Collection "Perovskites at the nanoscale: from fundamentals to applications", 8 (12), pp.6369-6378. 10.1039/C5NR07175E . hal-01242389

\section{HAL Id: hal-01242389 \\ https://hal.science/hal-01242389}

Submitted on 30 Nov 2016

HAL is a multi-disciplinary open access archive for the deposit and dissemination of scientific research documents, whether they are published or not. The documents may come from teaching and research institutions in France or abroad, or from public or private research centers.
L'archive ouverte pluridisciplinaire HAL, est destinée au dépôt et à la diffusion de documents scientifiques de niveau recherche, publiés ou non, émanant des établissements d'enseignement et de recherche français ou étrangers, des laboratoires publics ou privés. 


\title{
Quantum confinement and dielectric profiles of colloidal nanoplatelets of halide inorganic and hybrid organic-inorganic perovskites ${ }^{\dagger}$
}

\author{
Daniel Sapori, ${ }^{a}$ Mikaël Kepenekian, ${ }^{* b}$ Laurent Pedesseau, ${ }^{a}$ Claudine Katan, ${ }^{b}$ Jacky Even ${ }^{* a}$
}

\author{
Received Xth $X X X X X X X X X X 20 X X$, Accepted Xth $X X X X X X X X X 20 X X$ \\ First published on the web Xth $X X X X X X X X X X 200 X$ \\ DOI: $10.1039 / b 000000 x$
}

Quantum confinement as well as high frequency $\varepsilon_{\infty}$ and static $\varepsilon_{s}$ dielectric profiles are described for nanoplatelets of halide inorganic perovskites $\mathrm{CsPbX}_{3}(\mathrm{X}=\mathrm{I}, \mathrm{Br}, \mathrm{Cl})$ and hybrid organic-inorganic perovskites (HOP) in two-dimensional (2D) and three-dimensional (3D) structures. 3D HOP are currently sought for their impressive photovoltaic ability. Prior to this sudden popularity, 2D HOP materials were driving intense activity in the field of optoelectronics. Such developments have been enriched by the recent ability to synthesize colloidal nanostructures of controlled size of 2D and 3D HOP. This raises the need to achieve a thorough description of the electronic structure and dielectric properties of these systems. In this work, we go beyond the abrupt dielectric interface model and reach atomic scale description. We examine the influence of the nature of the halogen and of the cation on the band structure and dielectric constants. Similarly, we survey the effect of dimensionality and shape of the perovskite. In agreement with recent experimental results, we show an increase of the band gap and a decrease of $\varepsilon_{\infty}$ when the size of a nanoplatelet reduces. By inspecting 2D HOP, we find that it cannot be described as a simple superposition of independent inorganic and organic layers. Finally, the dramatic impact of ionic contributions on the dielectric constant $\varepsilon_{s}$ is analysed.

\section{Introduction}

Solution-processed three-dimensional (3D) halide perovskites of general formula $\mathrm{RMX}_{3}(\mathrm{M}=\mathrm{Pb}, \mathrm{Sn}, \mathrm{Ge}$ and $\mathrm{X}=\mathrm{I}, \mathrm{Br}$, $\mathrm{Cl}$, Fig. 1-a) have become, over the last three years, photovoltaics superstars, provided that the cation $\mathrm{R}$ is an organic cation (e.g. methylammonium $\mathrm{CH}_{3} \mathrm{NH}_{3}^{+}$). Indeed, hybrid organic-inorganic perovskites (HOP) based solar cells have shown power conversion efficiencies growing from $3.8 \%$ in $2009^{1}$ to over $20 \%$ in $2015 .^{2}$ In addition to impressive performances, HOP offer easy processability (low temperature, full solution process) and thus open the way towards low cost production of high-efficiency solar cells.

As far as chemistry is concerned, these materials offer a chemical playground. Depending on the choice of the metal, the anion or the organic cation, HOP crystallize in various structures. Thus, chemical engineering offers the possibility to tune properties such as band gaps. ${ }^{3,4}$ In particular, the structure is sensitive to the size of the organic cation. For molecular ions 'large enough' (e.g. $\left.\mathrm{C}_{4} \mathrm{H}_{9} \mathrm{NH}_{3}^{+}\right)$, the compound is

$\dagger$ Electronic Supplementary Information (ESI) available: Complementary results on the electronic structure and dielectric constants of $\mathrm{CsPbX}_{3}$ and $\mathrm{CH}_{3} \mathrm{NH}_{3} \mathrm{PbX}_{3}$ (X = I, Br, Cl). See DOI: 10.1039/b000000x/

a Université Européenne de Bretagne, INSA, FOTON UMR 6082, 35708 Rennes, France; E-mail: jacky.even@insa-rennes.fr

${ }^{b}$ Institut des Sciences Chimiques de Rennes, UMR 6226, CNRS - Université de Rennes 1, Campus de Beaulieu, 35042 Rennes, France; E-mail: mikael.kepenekian@univ-rennes1.fr (a)

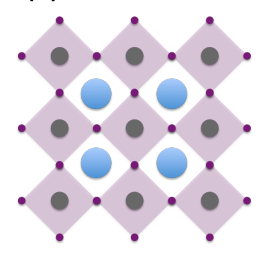

3D

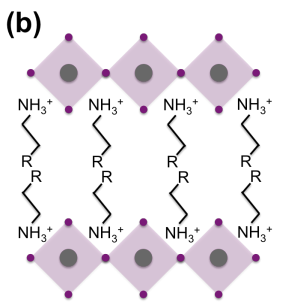

2D

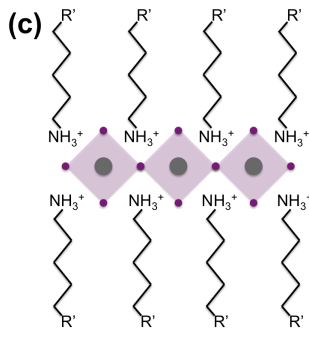

CNPL
Fig. 1 Schemes of (a) 3D halide perovskite, (b) 2D hybrid organic-inorganic halide perovskite, and (c) colloidal nanoplatelet of hybrid organic-inorganic halide perovskite. Grey circles stand for metal cations ( $\mathrm{Pb}, \mathrm{Sn}, \mathrm{Ge})$, purple circles for halides (I, $\mathrm{Br}, \mathrm{Cl})$, and blue circles for inorganic (Cs) or organic cations.

organized as a two-dimensional (2D) structure with an organic layer buffering inorganic slabs (Fig. 1-b). Incidentally, intense investigations on HOP started more than two decades ago when Mitzi and coworkers designed thin-film transistors based on 2D HOP. ${ }^{5,6}$ Up to 2009, solar cell applications, although anticipated, were not pursued and HOP became popular in the field of optoelectronics for production of transistors and lightemitting devices. ${ }^{7-11}$ In addition to these bulk $2 \mathrm{D}$ and 3D materials, nanostructures of perovskites, e.g. colloidal nanoplatelets (CNPL, Fig. 1-c), have recently been reported. ${ }^{12-20}$

The shape given to HOP has an impact on its excitonic prop- 
erties. 2D HOP are known to present a strong exciton binding energy $^{21}$ related to abrupt dielectric interfaces ${ }^{22}$ and exhibit a quantum confinement of type $\mathrm{I}^{23,24}$ with a dielectric confinement ${ }^{22,25}$ attributed to the contrast between the low dielectric constant of the organic part ${ }^{26}$ and the high dielectric constant of the inorganic part. ${ }^{27}$ On the other hand, 3D HOP show an exciton screened by polar vibrational modes and disordered configurations of polar organic cations. ${ }^{28-31}$ The acquired control over the shape of the active materials allows one to tune quantum and dielectric confinements. The later is a crucial point as it has a direct impact on the binding energy of the exciton ${ }^{32}$ and thus, influence the separation and the transport of charges. Quantum size effects of HOP nanoplatelets of different thicknesses were investigated experimentally and an increase of the energy gap and of the exciton binding energy was observed for the thinner nanoplatelets. ${ }^{20}$ Theoretical inspections can shed light on these effects. In a pioneer contribution, Muljarov et al. calculate the binding energies of excitons in HOP. ${ }^{33}$ This method takes into account the dielectric confinement but relies on parameters fitted to experimental data. Moreover, to avoid unphysical divergences related to abrupt dielectric interfaces, a transitional layer at the interface between organic and inorganic parts has to be considered. Recently, we proposed a different approach based on density functional theory (DFT) calculations to describe dielectric profiles of 2D systems. ${ }^{34}$ Our method takes advantage of the $a b$ initio description of the nanoplatelets and in particular of interfaces.

In this work, we use DFT calculations and subsequent treatments to describe the quantum and dielectric confinements in halide perovskite-based nanostructures. We start by recalling the main features of the method. Then, we describe the quantum confinement in 3D all-inorganic perovskites $\mathrm{CsPbX}_{3}(\mathrm{X}=$ $\mathrm{I}, \mathrm{Br}, \mathrm{Cl}$ ) nanoplatelets as well as the most studied 3D HOP $\mathrm{CH}_{3} \mathrm{NH}_{3} \mathrm{PbX}_{3}(\mathrm{X}=\mathrm{I}, \mathrm{Br})$. The confinement effects are compared to the one observed in $2 \mathrm{D}$ and intermediate $(2 \mathrm{D} / 3 \mathrm{D})$ materials. In all cases, the expected enlargement of the band gap is observed when going to thinner systems. Then we discuss static and high frequency dielectric profiles and find a dielectric confinement for systems where the inorganic layer is less than $30 \AA$. In agreement with recent experimental data, we show a decrease of the dielectric constant with decreasing nanoplatelets thicknesses.

\section{Methods}

\section{Computational details}

First-principles calculations are based on DFT as implemented in the SIESTA package. ${ }^{35,36}$ Calculations have been carried out with the GGA functional in the PBE form. ${ }^{37}$ Atoms are described by scalar relativistic norm-conserving TroullierMartins pseudopotentials, ${ }^{38}$ that include 14 valence electrons for $\mathrm{Pb}, 7$ for I, 5 for $\mathrm{N}, 4$ for $\mathrm{C}$ and 1 for $\mathrm{H}$. Valence wave functions are described through a basis set of finite-range numerical pseudoatomic orbitals. ${ }^{39}$ Structures relaxation and electronic structure calculations have been done using a double$\zeta$ polarized basis sets. ${ }^{39}$ The geometries were optimized until the forces were smaller than $0.01 \mathrm{eV} . \AA^{-1}$. The electronic structure was converged using a $1 \times 4 \times 4 k$-point sampling of the Brillouin zone. In all cases, an energy cutoff of $150 \mathrm{Ry}$ for real-space mesh size has been used. After construction, the atomic positions of the slabs of $\mathrm{CsPbX}, \mathrm{CH}_{3} \mathrm{NH}_{3} \mathrm{PbI}_{3}$ and $\mathrm{CH}_{3} \mathrm{NH}_{3} \mathrm{PbBr}_{3}$ are first relax (along the [010] direction) in the absence of any electric field. These optimized structures are then used as the starting point to compute the electronic and dielectric properties. The high frequency dielectric constants are obtained by relaxing the sole electron density under the external electric field, keeping the atomic positions fixed. To compute the static dielectric constant, the atomic positions are relaxed both in the absence and in the presence of the electric field. The choice of the magnitude of the external electric field is made based on a compromise between large enough variation of the electron density and absence of higher-order contributions (e.g. $\left.0.1 \mathrm{~V} . \AA^{-1}\right)$.

Let us note that spin-orbit coupling (SOC) is not taken into account in the present calculations. Although it is knows to be of importance for the description of the electronic structure of HOP (especially for lead-based HOP), it leads to significant underestimation of the band gap. ${ }^{25,40-44}$ In turn, this would lead to an artifactual overestimation of the dielectric constant and artifacts in the calculated profiles. Adding many-body corrections, such as in SOC+GW calculations, would provide an improved alternative. Unfortunately, this level of theory has a much larger computational cost, thereby preventing the investigation of layered structures or colloidal nanoplatelets.

\section{Dielectric profiles}

Despite the importance of the dielectric constant, its description often relies on a crude continuum modeling of the dielectric heterostructures by abrupt interfaces, with one dielectric constant value for the semiconductor core and another one for the surfaces and the surrounding medium. ${ }^{45-47}$ Alternative $A b$ initio-based approaches of interfaces have also been proposed, based on DFT calculations of free-standing silicon/oxyde and ferroelectric slabs ${ }^{48,49}$ or other heterostructures. ${ }^{50}$ These approaches rely on periodic supercells, a plane wave description of the wavefunction and are included in the self-consistent part of widely used first-principles codes such as VASP ${ }^{51}$ or Quantum ESPRESSO ${ }^{52}$ Next, taking advantage of the strictly localized basis set description available in the SIESTA code, ${ }^{35,36}$ offers another type of approach to the determination of dielectric profiles (see ESI $\dagger$ ). ${ }^{34,53,54}$ Such an approach is computationally cheaper, takes full advantage of the costless representation of 
vacuum and is parameter free. Here we recall the main points of the latter method, more details can be found in Ref. 34 .

Similarly to the scheme introduced by Giustino and coworkers, ${ }^{55}$ dielectric profiles are obtained without the description of the whole dielectric matrix, thus reducing the computational time. The induced variation of the planar averaged electron density, $\delta \rho_{\text {ind }}(z)$, is given by $\delta \rho_{\text {ind }}(z)=\left.\rho(z)\right|_{\vec{E}_{\text {ext }}}-\left.\rho(z)\right|_{0}$, where $\vec{E}_{\text {ext }}$ is an external electric field. Longitudinal nanoscale and transverse macroscopic averages are performed to derive nanoscale dielectric properties. ${ }^{34,53,54}$ This is important to ensure proper connection with the macroscopic dielectric constant of the bulk material.

Applying an electric field is performed on slabs which are periodically reproduced. Thus, the induced polarization shall be corrected for unphysical inter-slab interactions. This cannot be performed directly on the induced polarization. A trick is to calculate the total induced dipole $m_{z}(L)$ for various interslab distances $L$ with and without the external electric field. The slope of the inverse of $m_{z}(L)$ as a function of $1 / L$ is equal to $1 /\left(\varepsilon_{0} . E_{\text {ext }}\right)$, where $\varepsilon_{0}$ is the vacuum permittivity. ${ }^{53}$ The induced dipole $m_{z}(L \rightarrow \infty)$ for an infinite $L$, is simply obtained by extrapolating $1 / L$ to 0 . The ratio $m_{z}(L \rightarrow \infty) / m_{z}(L)$ is then employed to correct the induced polarization from the artificial interaction between the slabs.

Four steps are required to derive the dielectric constant profiles along the stacking axis of the slab from the calculated DFT electron density:

(i) The electron density has first to be averaged in the plane perpendicular to the stacking axis. The electron density profile $\rho(z)$ is then smoothed using a nanoscale averaging along the stacking axis. ${ }^{56}$ The length of the filter function is chosen from the study of the Hartree potential profile. For perovskites investigated in this works, it is equal to the distance between two adjacent octahedra. This is in good agreement with the definition of macroscopic internal fields in a solid (vide infra).

(ii) Compute the induced electron density from the DFT computations with and without an external electric field: $\delta \rho_{\text {ind }}(z)=\left.\rho(z)\right|_{\vec{E}_{\text {ext }}}-\left.\rho(z)\right|_{0}$.

(iii) Compute the induced polarization $p_{\text {ind }}(z)$ by partial integration of the induced electron density

$$
\frac{d p_{\text {ind }}(z)}{d z}=-\delta \rho_{\text {ind }}(z)
$$

and correct for the unphysical inter-slab interaction using the ratio $m_{z}(L \rightarrow \infty) / m_{z}(L)$. The total induced dipole is given by

$$
m_{z}=\int_{-\infty}^{z} p_{\text {ind }}(z) d z \text {. }
$$

(iv) Derive the nanoscopic average of the dielectric constant profile $\varepsilon(z)$ from the induced polarization

$$
\varepsilon_{\infty}(z)=\frac{\varepsilon_{0} E_{\text {ext }}}{\varepsilon_{0} E_{\text {ext }}-p_{\text {ind }}(z)} .
$$

The dielectric constant depends on the frequency of the electric field, the temperature and the direction. It is usually describe by a tensor when the system is anisotropic. In the high frequency limit $\left(\varepsilon_{\infty}\right)$, it takes into account only the distortion of the electron density by the electric field. For lower frequencies $\left(\varepsilon_{s}\right)$ it takes into account both electronic and ionic effects (phonons). For extremely low frequencies, other interactions such as dipolar interactions contribute as well. In the present work, the latter are not included in our computation of $\varepsilon_{s}$. Moreover, as we use a static electric field, we are bound to the description of limit cases. The inspection of intermediate regimes would involve the use of time-dependent electric field and of more sophisticated approaches that are generally dedicated to the study of laser-irradiated materials. ${ }^{57-61}$

\section{Investigated structures}

First, we consider the all-inorganic perovskites $\mathrm{CsPbX}_{3}(\mathrm{X}=\mathrm{I}$, $\mathrm{Br}, \mathrm{Cl})$ series. Recently, synthesis of colloidal nanocrystals and nanoplatelets of these compounds has been reported. ${ }^{17,62}$ Starting from the $P m \overline{3} m$ cubic phase (no. 221), ${ }^{63}$ we construct slabs with thicknesses going from 1 to 8 layers, each layer consisting in an octahedra $\mathrm{PbI}_{6}$ stacked in the [001] direction (Fig. 2-a). Similar slabs are constructed from the low-temperature Pnma (no. 62) phase of $\mathrm{CH}_{3} \mathrm{NH}_{3} \mathrm{PbI}_{3}$ and $\mathrm{CH}_{3} \mathrm{NH}_{3} \mathrm{PbBr}_{3}$ (Fig. 2b) ${ }^{64,69}$ in the [010], [100] and [001] direction.

A series of 2D HOP are then explored (Fig. 2-c-e). In those systems a one-octahedron thick inorganic layer is sandwiched between layers of organic cations. The effect of the molecule on the confinement is inspected. The organic cation is aromatic for $\left(\mathrm{C}_{7} \mathrm{H}_{10} \mathrm{~N}\right)_{2} \mathrm{PbI}_{4},{ }^{65}$ aliphatic for $\left(\mathrm{C}_{4} \mathrm{H}_{12} \mathrm{~N}\right)_{2} \mathrm{PbI}_{4}$ and $\left(\mathrm{C}_{10} \mathrm{H}_{24} \mathrm{~N}\right)_{2} \mathrm{PbI}_{4}{ }^{66,67}$ Finally, the 2D/3D $\mathrm{HOP}\left(\mathrm{C}_{10} \mathrm{H}_{24} \mathrm{~N}\right)_{2} \mathrm{PbI}_{3} \mathrm{CH}_{3} \mathrm{NH}_{3} \mathrm{PbI}_{4}$ is investigated (Fig. 2-f). ${ }^{68}$ In this system, the inorganic layer is 2-octahedron thick.

\section{Results and discussion}

\section{Quantum confinement in nanoplatelets}

We first stress that it is well known that semiconductor band gaps are poorly reproduced by plain DFT calculations (vide infra). However, the qualitative trends are well reproduced and comparisons between similar structures can be conducted.

Bulk $\mathrm{CsPbX}_{3}(\mathrm{X}=\mathrm{I}, \mathrm{Br}, \mathrm{Cl})$ in the $P m \overline{3} m$ phase presents a direct band gap at $\mathrm{R}$ (Fig. S1, ESI $\dagger$ ). ${ }^{70-72}$ The main impact climbing down the halogen column is the reduction of the band gap that goes from $2.54 \mathrm{eV}$ for $\mathrm{CsPbCl}_{3}$ to $1.73 \mathrm{eV}$ for $\mathrm{CsPbI}_{3}$ 


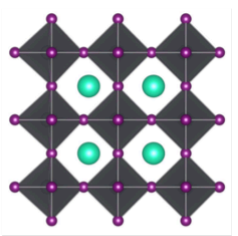

(a)

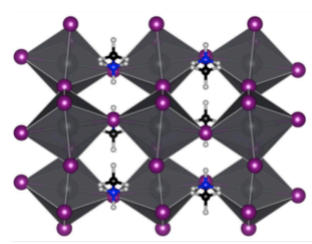

(b)

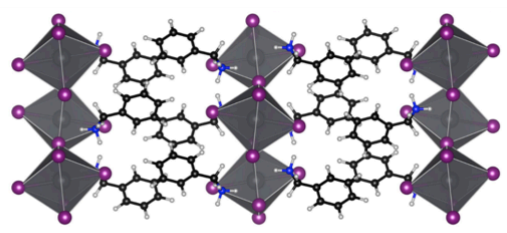

(c)

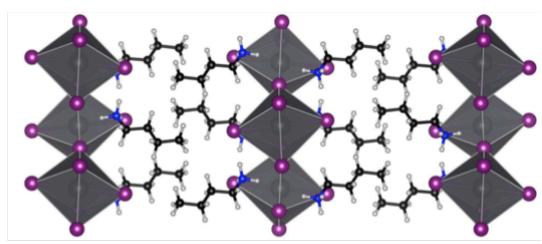

(d)

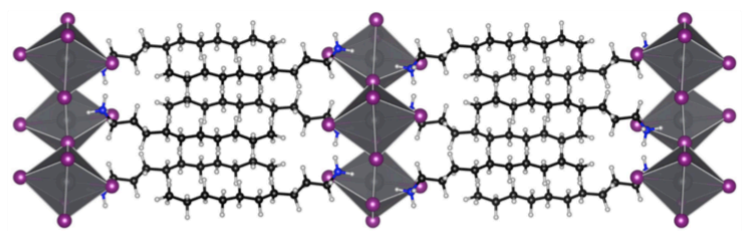

(e)

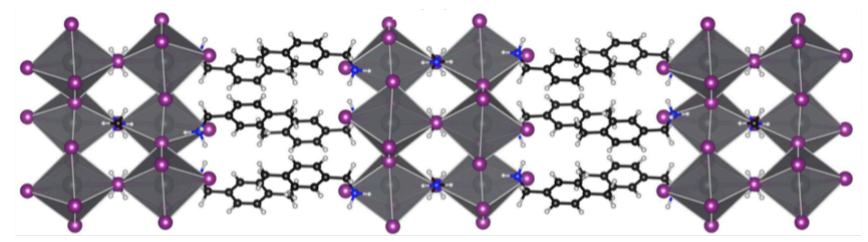

(f)

Fig. 2 Structures of (a) all-inorganic $\mathrm{CsPbX}_{3}(\mathrm{X}=\mathrm{I}, \mathrm{Br}, \mathrm{Cl})$ in the $P m \overline{3} m$ phase, ${ }^{63}$ (b) the $3 \mathrm{D}$ HOP $\mathrm{CH}_{3} \mathrm{NH}_{3} \mathrm{PbI}_{3}$ in the low-temperature Pnma phase, ${ }^{64}$ a series of $2 \mathrm{D}$ HOP with (c) aromatic organic cation $\left(\mathrm{C}_{7} \mathrm{H}_{10} \mathrm{~N}\right)_{2} \mathrm{PbI}_{4},{ }^{65}$ (d) and (e) aliphatic organic cation $\left(\mathrm{C}_{4} \mathrm{H}_{12} \mathrm{~N}\right)_{2} \mathrm{PbI}_{4}$, and $\left(\mathrm{C}_{10} \mathrm{H}_{24} \mathrm{~N}\right)_{2} \mathrm{PbI}_{4},{ }^{66,67}$ respectively and (f) the $2 \mathrm{D} / 3 \mathrm{D}-\mathrm{HOP}$ $\left(\mathrm{C}_{10} \mathrm{H}_{24} \mathrm{~N}\right)_{2} \mathrm{PbI}_{3} \mathrm{CH}_{3} \mathrm{NH}_{3} \mathrm{PbI}_{4} .{ }^{68} \mathrm{~Pb}, \mathrm{Cs}, \mathrm{I}, \mathrm{N}, \mathrm{C}$ and $\mathrm{H}$ atoms are depicted in gray, green, purple, blue, black and white, respectively.

(Table 1). When going to slabs of $\mathrm{CsPbX}_{3}$, the cubic symmetry is lost and the $\mathrm{R}$ and $\mathrm{M}$ points become equivalent. Fig. 3-a presents the band structure of $\mathrm{CsPbI}_{3}$ slabs for various thicknesses. The expected behavior is retrieved when going to thinner slabs: the band gap increases from $1.79 \mathrm{eV}$ to $1.94 \mathrm{eV}$ under the effect of quantum confinement (Fig. 3-b). For a 8-layer slab, the bulk band gap is nearly recovered, i.e. the confinement vanishes. The relative increase of the band gap due to quantum confinement becomes slightly larger for $\mathrm{Br}$ and $\mathrm{Cl}$ based perovskites (Table 1 and Fig. S2, ESI $\dagger$ ) with band gaps varying from 2.11 to $2.32 \mathrm{eV}$ and 2.50 to $2.75 \mathrm{eV}$, respectively. These results are consistent with recent experimental data reporting an increase of the band gap energy with the reduction of the semiconductor $\mathrm{CsPbX}_{3}$ core of the QD. ${ }^{62}$
Table 1 Calculated band gap (eV) for bulk and slabs of all inorganic and organic-inorganic perovskites.

\begin{tabular}{lccccccc}
\hline \hline Cation & & \multicolumn{3}{c}{$\mathrm{Cs}^{+}$} & & \multicolumn{2}{c}{$\mathrm{CH}_{3} \mathrm{NH}_{3}^{+}$} \\
\cline { 7 - 8 } \cline { 7 - 8 } Halogen & & $\mathrm{I}$ & $\mathrm{Br}$ & $\mathrm{Cl}$ & & $\mathrm{I}$ & $\mathrm{Br}$ \\
\hline Bulk & & 1.73 & 2.11 & 2.54 & & 2.05 & 2.50 \\
8-layer & & 1.79 & 2.11 & 2.50 & & 2.07 & 2.44 \\
4-layer & & 1.85 & 2.19 & 2.60 & & 2.15 & 2.51 \\
2-layer & & 1.94 & 2.27 & 2.68 & & 2.31 & 2.60 \\
1-layer & & 1.93 & 2.32 & 2.75 & & 2.48 & 2.77 \\
\hline \hline
\end{tabular}

With the organic cation $\mathrm{CH}_{3} \mathrm{NH}_{3}^{+}$, the band structure of nanoplatelets (Fig. 4-a) shows a direct band gap at the $\Gamma$ point, as in the corresponding Pnma bulk. ${ }^{11,40,43,44,73}$ Again, when decreasing the size of the slab, the band gap increases, going from $2.07 \mathrm{eV}$ for the 8 -layer slab to $2.48 \mathrm{eV}$ for the 1-layer slab. The effect of quantum confinement becomes significant for slabs formed of less than 4 layers (Table 1 and Fig. 4-b). Slabs of $\mathrm{CH}_{3} \mathrm{NH}_{3} \mathrm{PbBr}_{3}$ follow the same trend (Fig. S3, ESI $\dagger$ ) with band gaps varying from 2.44 to $2.77 \mathrm{eV}$.

The variation of the band gap of all-inorganic and hybrid perovskite slabs with the number of layers $\mathrm{N}$ can be expressed as

$$
E_{g}(N)=E_{g, b u l k}+\delta E_{g}(N),
$$

where $E_{g}(N)$ is the band gap of the bulk core material and $\delta E_{g}(N)$ stems from quantum and dielectric confinement effects.

The various contributions can be estimated from theoretical calculations by using the following decomposition:

$$
E_{g}(N)=E_{g, D F T, b u l k}+\delta E_{g, D F T}(N)+\Sigma_{b u l k}+\delta \Sigma(N),
$$

where $E_{g, D F T, b u l k}$ and $\Sigma_{\text {bulk }}$ are respectively the bulk band gap evaluated at the DFT level and the self-energy corrections due to many-body effects. The self-energy corrections are very often computed with the GW approximation. For lead-based perovskites, the various contributions should in principle include SOC effects.

The effect of quantum confinement can be estimated from $\delta E_{g, D F T}(N)$, provided that the effective masses of the bulk materials are accurately described by plain DFT calculations. In the case of $\mathrm{CH}_{3} \mathrm{NH}_{3} \mathrm{PbI}_{3}$, due to error cancellation, the DFT electronic band gap and effective masses are fortuitously in quite good agreement with $\mathrm{GW}+\mathrm{SOC}$ values. For this reason, the $\delta E_{g, D F T}(N)$ computed without SOC (Fig. 4) yield good estimates of the effect of quantum confinement.

Due to limited available computational power, the variation of the self-energy with the number of layers, $\delta \Sigma(N)$, cannot be evaluated directly from GW computations. An alternative semi classical evaluation of this self-energy correction will later on be given within the framework of the dielectric confinement theory (vide infra). 


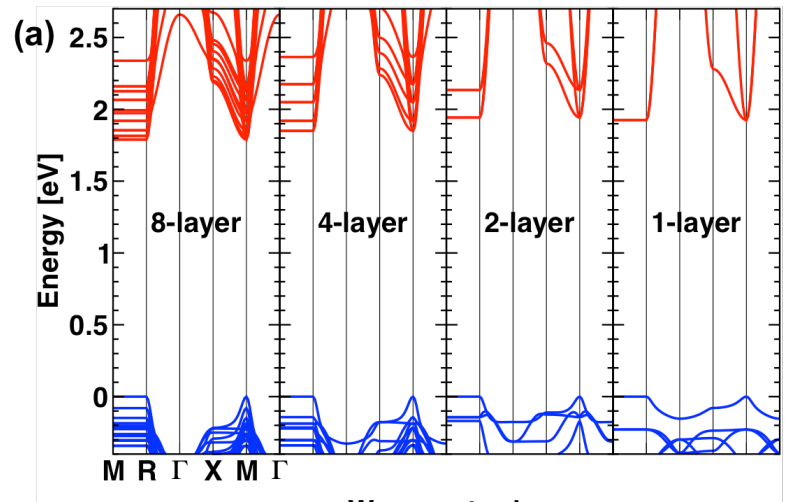

(b)

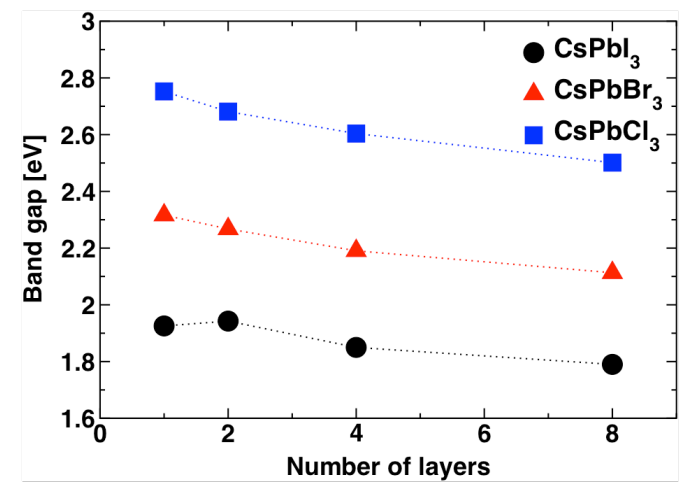

Fig. 3 (a) Band structures for slabs of $\mathrm{CsPbI}_{3}$. (b) Band gaps with respect to the slab thickness for $\mathrm{CsPbX}_{3}(\mathrm{X}=\mathrm{I}, \mathrm{Br}, \mathrm{Cl})$.

Experimentally, such effects were observed for $\mathrm{CH}_{3} \mathrm{NH}_{3} \mathrm{PbI}_{3}$ and $\mathrm{CH}_{3} \mathrm{NH}_{3} \mathrm{PbBr}_{3}$ nanoobjects. ${ }^{15,16,20}$ However, Sichert et al. notice that for colloidal nanoplatelets the optical band gap evolution does not show the sudden variation anticipated by various models and also observed in our DFT results. ${ }^{20}$ This supports an increase of the exciton binding energy in thinner slabs that may compensate the expected band gap enlargement related to quantum confinement. It prompts further inspection of dielectric confinement in such structures.

The behavior of the all-inorganic and hybrid perovskites nanoplatelets are qualitatively similar with respect to quantum confinement. As expected, the band gap increases when the thickness of the slab decreases. This effect gets stronger when the halogen is lighter $(\mathrm{Cl}>\mathrm{Br}>\mathrm{I})$. However, all these observations are much more pronounced in the case of an organic cations that emphasize quantum confinement.

\section{High frequency dielectric profile}

The high dielectric profiles $\varepsilon_{\infty}(z)$ of $\mathrm{CsPbI}_{3}$ is depicted in Fig. 5-a. In the case of a large slab (8 layers), the bulk dielectric constant is recovered at its center amounting to 5.0. The same is observed for $\mathrm{CsPbBr}_{3}$ and $\mathrm{CsPbCl}_{3}$ (Fig. S4, ESI $\dagger$ )

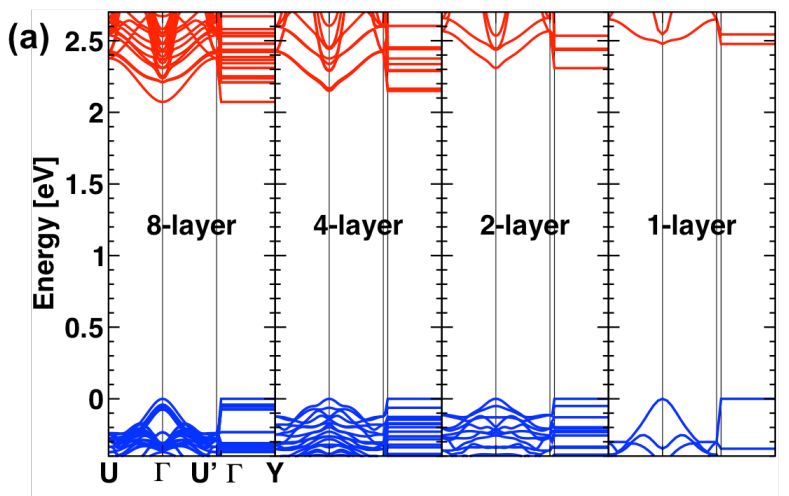

Wavevector $\mathbf{k}$

(b)

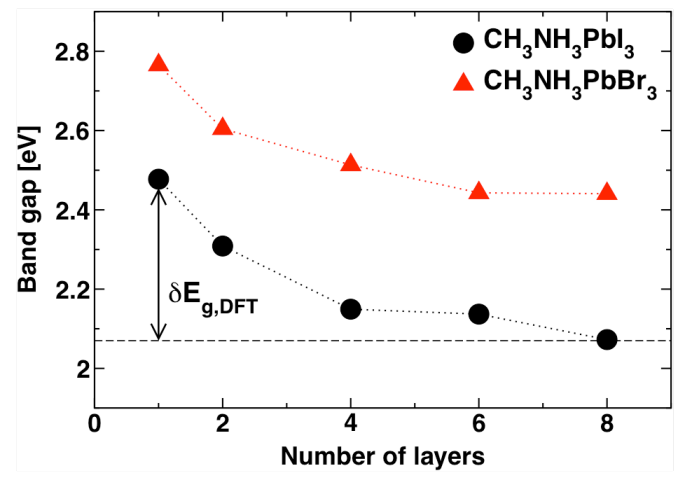

Fig. 4 (a) Band structures for slabs of $\mathrm{CH}_{3} \mathrm{NH}_{3} \mathrm{PbI}_{3}$ with various thicknesses. (b) Band gaps with respect to the slab thickness for $\mathrm{CH}_{3} \mathrm{NH}_{3} \mathrm{PbX}_{3}(\mathrm{X}=\mathrm{I}, \mathrm{Br})$.

with bulk-like dielectric constants of 3.8 and 3.2, respectively. In the case of $\mathrm{CsPbCl}_{3}$, the dielectric constant has experimentally been determined and ranges between 3.6 and 3.7, ${ }^{74}$ in fair agreement with our computed value. The reduction of the slab thickness leads to a decrease of the dielectric constant (Fig. 5-a and Fig. 6) that tends to converge to the medium value 1. Dielectric properties significantly different from those of the bulk material are observed for slabs of less than 4 layers with a more pronounced variation when going from chlorine to iodine.

The substitution of $\mathrm{Cs}^{+}$by the organic cation $\mathrm{CH}_{3} \mathrm{NH}_{3}^{+}$in the 3D HOP structure leads to an enhancement of the dielectric constant (Fig. 5-b). For slabs constructed in the [010] direction, the dielectric constant at the center converges to 5.6 for thick enough slabs. The low temperature Pnma phase is not isotropic and one has to inspect all directions. We find that the dielectric constant reaches 4.9 and 4.8 for slabs oriented along [100] and [001], respectively (Fig. S5, ESI $\dagger$ ). The average value $\overline{\varepsilon_{\infty}}=5.1$, compares well with the experimental value of 6.5 measured on the Pmma phase ${ }^{75}$ and to previous values obtained by DFT+SOC for different orientations of the cation ${ }^{76}$ or $\mathrm{GW}+\mathrm{SOC}$ calculations. ${ }^{42}$ For $\mathrm{CH}_{3} \mathrm{NH}_{3} \mathrm{PbBr}_{3}$, a bulk value of 4.2 is found. One can notice that the halogen 


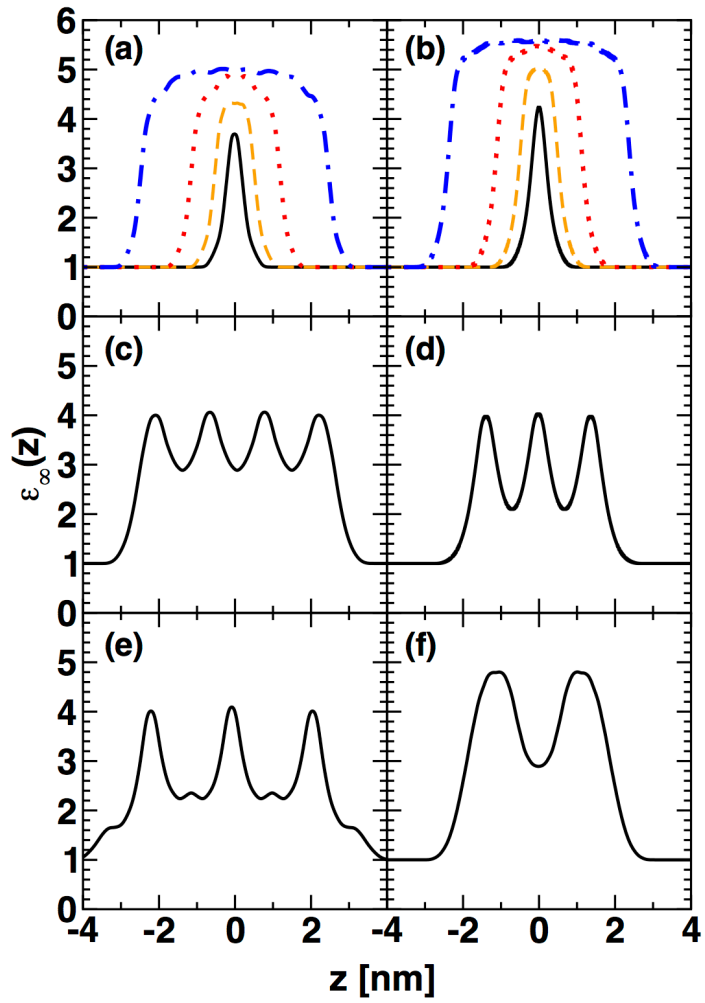

Fig. 5 High frequency dielectric profiles $\varepsilon_{\infty}$ (z) for slabs of (a) $\mathrm{CsPb}_{3}$, (b) $\mathrm{CH}_{3} \mathrm{NH}_{3} \mathrm{PbI}_{3}$ (in the [010] direction). Black, orange, red and blue lines correspond to 1, 2, 4 and 8-layer slabs, respectively. $\varepsilon_{\infty}(\mathrm{z})$ for (c) the $2 \mathrm{D}$ HOP with aromatic cation $\left.\mathrm{C}_{7} \mathrm{H}_{10} \mathrm{~N}\right)_{2} \mathrm{PbI}_{4}$, the 2D HOP with aliphatic cations (d) $\left(\mathrm{C}_{4} \mathrm{H}_{12} \mathrm{~N}\right)_{2} \mathrm{PbI}_{4}$, and (e) $\left(\mathrm{C}_{10} \mathrm{H}_{24} \mathrm{~N}\right)_{2} \mathrm{PbI}_{4}$. (f) Same for the 2D/3D HOP $\left(\mathrm{C}_{10} \mathrm{H}_{24} \mathrm{~N}\right)_{2} \mathrm{PbI}_{3} \mathrm{CH}_{3} \mathrm{NH}_{3} \mathrm{PbI}_{4}$.

influence remains the same whatever the nature of the cation $\left(\varepsilon_{\infty}^{C s P b B r_{3}}(0) / \varepsilon_{\infty}^{C s P b I_{3}}(0) \approx \varepsilon_{\infty}^{\mathrm{CH}_{3} \mathrm{NH}_{3} \mathrm{PbBr}_{3}}(0) / \varepsilon_{\infty}^{\mathrm{CH}_{3} \mathrm{NH}_{3} \mathrm{PbI}_{3}}(0)\right)$.

As in the all-inorganic materials, the size reduction of $\mathrm{CH}_{3} \mathrm{NH}_{3} \mathrm{PbI}_{3}$ nanoplatelets shows a dielectric confinement for slabs of less than 4 layers, resulting in a $\varepsilon_{\infty}(0)$ of 4.2 for the 1-layer platelet (Fig. 5-b and Fig. 6). The same is observed with slabs of $\mathrm{CH}_{3} \mathrm{NH}_{3} \mathrm{PbBr}_{3}$ (Fig. 6 and Fig. S6, ESI $\dagger$ ) with value going from 4.2 to 3.3 for the thinnest nanoplatelet. These results indicate that little or vanishing dielectric confinement is expected for all-inorganic perovskites $\mathrm{CsPbX}_{3}$ or HOP $\mathrm{CH}_{3} \mathrm{NH}_{3} \mathrm{PbX}_{3}(\mathrm{X}=\mathrm{I}, \mathrm{Br}, \mathrm{Cl})$ with thicknesses larger than 4 layers.

In the case of 2D HOP, the profiles of the high frequency dielectric constant present a clear dielectric contrast between the low dielectric constant of the organic part and the high dielectric constant of the inorganic part (Fig. 5-c to e). For all 2D materials, the value obtained for the inorganic part is around 4 (Table S2, ESI $\dagger$ ). As expected, this value is lower than the bulk value of $3 \mathrm{D}$ materials (e.g. $\mathrm{CH}_{3} \mathrm{NH}_{3} \mathrm{PbI}_{3}$ ) and closer to

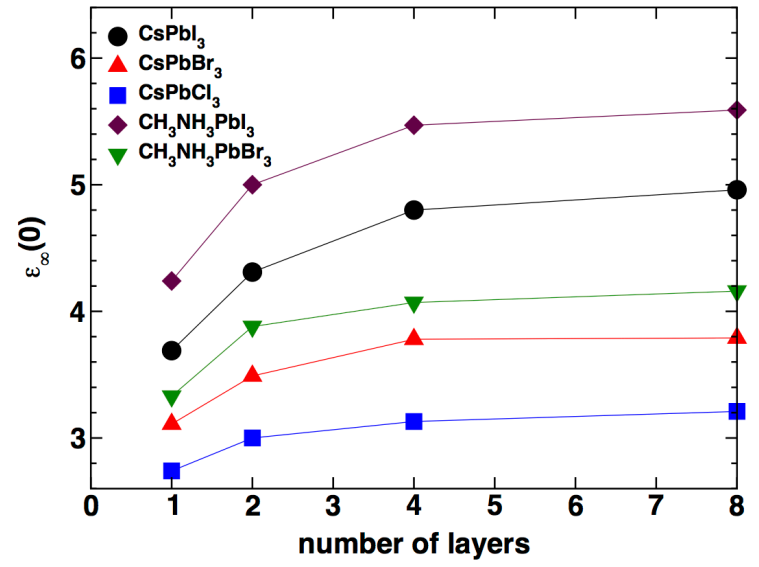

Fig. 6 High frequency dielectric constant at the slab centre $\varepsilon_{\infty}(0)$ for $\mathrm{CsPbX}_{3}(\mathrm{X}=\mathrm{I}, \mathrm{Br}, \mathrm{Cl})$ and $\mathrm{CH}_{3} \mathrm{NH}_{3} \mathrm{PbX}_{3}(\mathrm{X}=\mathrm{I}, \mathrm{Br})$ in the [010] direction.

the value obtained for 1-layer slabs of 3D HOP (Fig. 5-b, Table S1, ESI $\dagger$ ). The dielectric constant obtained for the aromatic cation (Fig. 5-c) amounts to 2.9 and is higher than the one obtained for the aliphatic chains, 2.1 (Fig. 5-d and e). The later is in agreement with the experimental high frequency dielectric constant reported to be about 2.2-2.3. ${ }^{26}$ Thus, if the size of the aliphatic chain affects the extent of the low dielectric constant zone, it does not seem to modify significantly the value of the constant itself.

Finally, Fig. 5-f shows the dielectric profile of the 2D/3D compound $\left(\mathrm{C}_{10} \mathrm{H}_{24} \mathrm{~N}\right)_{2} \mathrm{PbI}_{3} \mathrm{CH}_{3} \mathrm{NH}_{3} \mathrm{PbI}_{4}$. Interestingly, the dielectric constant in the inorganic layer amounts to 4.8 , which lies in between the value calculated for the purely 3D and purely $2 \mathrm{D}$ bulk systems. As in the 2D cases discussed above, the value is close, but slightly lower, than the value computed for a 2-layer platelet of $\mathrm{CH}_{3} \mathrm{NH}_{3} \mathrm{PbI}_{3}$ (Fig. 5-b).

From our results, the nature of the cation (inorganic $v s$. organic) has little influence on the high frequency dielectric profiles of 3D perovksites. Indeed, the amplitude of the confinement effect is of the same order even if the dielectric constant is about 1.2 times larger. For both cases, specific dielectric properties are observed for nanoplatelets of less than 4 layers. In addition, similar effects show up in the inorganic layers of 2D HOP.

\section{Static dielectric profile}

When the frequency of the electric field becomes lower, atoms can move and participate to the polarization of the slab. Thus, an ionic contribution to the dielectric constant has to be taken into account. For a low frequency dependent electric field, it corresponds to the additional contribution of the polar phonons. Here, the electric field is applied in the stacking direction of 
the slab. Fig. 7 shows the atomic displacement caused by an electric field on a $\mathrm{CH}_{3} \mathrm{NH}_{3} \mathrm{PbI}_{3}$ slab. It highlights the shrinking and stretching of lead-iodine bonds in the staking direction, which correspond to the main electric field induced distortions. Under electric field, halogen-lead bonds alternate between long

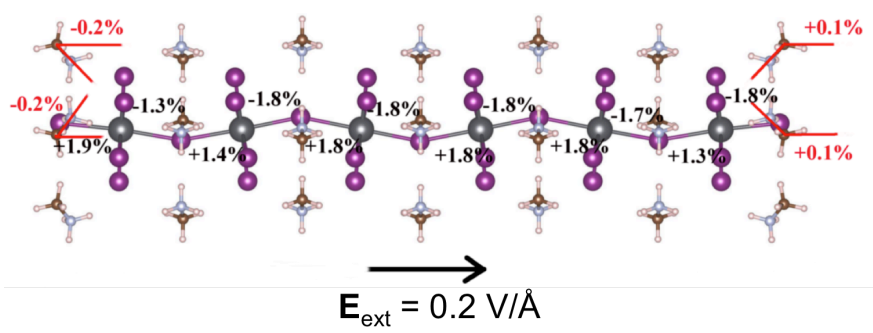

Fig. 7 Modification of the I-Pb bonds in a 6-layer of $\mathrm{CH}_{3} \mathrm{NH}_{3} \mathrm{PbI}_{3}$ slab induced by an electric field.

and short. Concomitantly, an asymmetry shows up, and more specifically a loss of inversion symmetry. Therefore, each octahedron bears an induced dipole that participates to the global dipole and polarization of the slab. This can be related to the bulk phonon contribution. In addition, organic cations at surfaces are free to rotate and therefore naturally align with the electric field, leading to a specific contribution from surface phonons.

The dielectric profiles $\varepsilon_{s}(z)$ of 6-layer and 1-layer slabs of $\mathrm{CsPbI}_{3}$ (Fig. 8-a) show the dramatic impact of the ionic contribution on the dielectric constant. At the center of the 6-layer slab, it gets multiplied by a factor of 7 increasing from $\varepsilon_{\infty}=5.0$ to a value oscillating around 35 . Large surface effects can be observed with the inorganic cation $\mathrm{Cs}^{+}$and $\varepsilon_{s}$ reaches values beyond 60. Confinement leads to a strong reduction of $\varepsilon_{s}$ to 22.6 for the thinnest nanoplatelet.

When replacing the inorganic cation by an organic cation, for instance $\mathrm{CH}_{3} \mathrm{NH}_{3}^{+}$, the dielectric profile of $\varepsilon_{s}$ becomes visibly smoother (Fig. 8-b). In fact, the surface coating by organic moieties cancels the abrupt increase of the dielectric constant near the surface. A similar behavior has been observed with CdSe CNPL passivated by acetates. ${ }^{34}$ The surface organic cation are reoriented under the effect of the external electric field and tend to screen its effects. As a consequence, the dielectric constant does no more endure a seven-fold increase but a four-fold growth from $\varepsilon_{\infty}=5.6$ to $\varepsilon_{s}=22.0$ at the centre of the 6-layer slab (in the [010] direction). Unfortunately, comparison to experimental results is difficult. In fact, various experimental values have been reported for the static (low) frequency dielectric constant of bulk $\mathrm{CH}_{3} \mathrm{NH}_{3} \mathrm{PbI}_{3}$, which depend on the sample (type/quality), temperature and on the method chosen: $28.8,{ }^{77} 23.3,{ }^{78}$ and $35^{79}$ for the tetragonal phase at room temperature and 36 for the orthorhombic phase. ${ }^{78}$ However, we find a better agreement with the increase of the reported bulk values of $\varepsilon$ when the ionic displacements start having signifi-

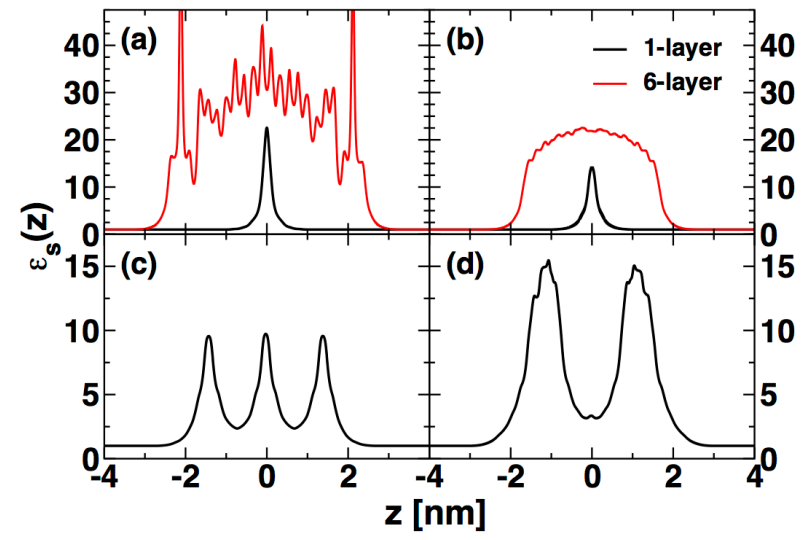

Fig. 8 Static dielectric profile $\varepsilon_{s}(\mathrm{z})$ for slabs of (a) $\mathrm{CsPbI}_{3}$ and (b) $\mathrm{CH}_{3} \mathrm{NH}_{3} \mathrm{PbI}_{3}$ (in the [010] direction). Black and red lines correspond to 1 and 6-layer slabs, respectively. $\varepsilon_{s}(\mathrm{z})$ for (c) the 2D HOP with aliphatic cations $\left(\mathrm{C}_{4} \mathrm{H}_{12} \mathrm{~N}\right)_{2} \mathrm{PbI}_{4}$, and (d) the 2D/3D HOP $\left(\mathrm{C}_{10} \mathrm{H}_{24} \mathrm{~N}\right)_{2} \mathrm{PbI}_{3} \mathrm{CH}_{3} \mathrm{NH}_{3} \mathrm{PbI}_{4}$.

cant contributions to the dielectric constant of the slab. When the confinement is extreme (1-layer slab), the dielectric constant is essentially dominated by surface effects. At the same time, it still shows significant increase as compared to its high frequency value (Fig. 5-b), but reduces to 14.1 (Fig. 8-b)

The static dielectric profile of the 2D HOP $\left(\mathrm{C}_{4} \mathrm{H}_{12} \mathrm{~N}\right)_{2} \mathrm{PbI}_{4}$ shows a remarkable feature (Fig. 8-c). The dielectric constant of the organic layer is roughly speaking not affected by the ionic contribution $\left(\varepsilon_{\infty}=2.1\right.$ vs. $\left.\varepsilon_{s}=2.4\right)$. On the other hand, the dielectric constant of the inorganic part is 2.5 times larger in the static regime $\left(\varepsilon_{s}=9.5\right)$ than in the high frequency one $\left(\varepsilon_{\infty}=3.9\right)$. It traces back to the anisotropy of $2 \mathrm{D}$ materials with the organic part that softens the displacement of the inorganic part. This reinforces the idea that 2D HOP are composite materials with specific properties ${ }^{24}$ including mechanical properties with region of hard and soft elastic constants. Besides, the maximum value obtained for this $2 \mathrm{D}$ material is much lower than the value obtained for the 1-layer slab of the 3D HOP (Fig. 8-b). The organic moieties are indeed different. But, as it had already been stressed for quantum confinement, ${ }^{24}$, in real 2D HOP structures the inorganic layer cannot be solely approximated by a single standalone layer derived from the $3 \mathrm{D}$ material.

A similar behavior is obtained for the 2D/3D HOP $\left(\mathrm{C}_{10} \mathrm{H}_{24} \mathrm{~N}\right)_{2} \mathrm{PbI}_{3} \mathrm{CH}_{3} \mathrm{NH}_{3} \mathrm{PbI}_{4}$ (Fig. 8-d). The dielectric constant of the inorganic part is around 15, showing a great enhancement with respect to the high frequency value $\varepsilon_{\infty}=4.8$ (Fig. 5-f). As in the case of 2D HOP, the motion of atoms changes only modestly the dielectric constant of the organic layer $\varepsilon_{s}=3.3 v s . \varepsilon_{\infty}=2.9$. 


\section{Dielectric confinement}

In layered heterostructures and slabs, the classical evaluation of the dielectric self-energy correction is related to a self-energy profile:

$$
\delta \Sigma\left(z_{0}\right)=\frac{e_{0}}{4 \pi} \int_{0}^{\infty}\left(V\left(q, z, z_{0}\right)-V_{\text {bulk }}\left(q, z, z_{0}\right)\right)_{z \rightarrow z_{0}} q \mathrm{~d} q .
$$

where $\mathrm{e}_{0}$ is a test charge at position $\mathrm{z}_{0} \cdot \delta \Sigma\left(z_{0}\right)$ is equivalent to the one particle potential profile acting on the test charge. The electrostatic potential felt by a particle located at $\mathrm{z}_{0}$ in a layered structure is computed by solving the inhomogeneous Poisson equation including the dielectric constant profile $\varepsilon(z)$ :

$$
\frac{\partial}{\partial z}\left(\varepsilon(z) \frac{\partial}{\partial z}\left(V\left(q, z, z_{0}\right)\right)\right)-q^{2} \varepsilon(z) V\left(q, z, z_{0}\right)=-e_{0} \delta\left(z-z_{0}\right) .
$$

The self-energy profile $\delta \Sigma\left(z_{0}\right)$ is plotted in Fig. 9-a for various slabs of $\mathrm{CH}_{3} \mathrm{NH}_{3} \mathrm{PbI}_{3}$. The shapes of the self-energy profiles are consistent with our previous study on colloidal nanoplatelets of II-VI semiconductors, where maximum values are observed at the interfaces between the platelets and vacuum. ${ }^{34}$ Moreover, the value of the self-energy increases at the center of the slab with decreasing thickness: this corresponds to the dielectric confinement effects. In addition, a semi-classical evaluation of the self-energy correction to the electronic band gap can be obtained, both for the $\mathrm{CB}$ and the VB states, by computing the following integrals:

$$
\delta \Sigma_{C B(V B)}(N)=\int \delta \Sigma(z, N) \rho_{C B(V B)}(z, N) \mathrm{d} z
$$

where $\rho_{C B(V B)}$ is the electronic density profile for the CB (VB) state. The sum of these two terms undergoes a steep increases for very thin slabs. This effect can be estimated by plotting the value of the self-energy at the center of the slab as a function of the number of layers (Fig. 9-b). We can thus conclude that the dielectric confinement effect is very important up to four layers.

Finally, we shall point out that the actual band gap obtained from optical absorption measurements, may undergo a smoother variation than the band gap predicted from the computation of monoelectronic states. Indeed, the optical band gap corresponds to the electronic band gap minus the exciton binding energy. The exciton binding energy increases, when the thickness decreases, which partially compensates the gap increase related to quantum and dielectric confinement effects (vide supra) on the monoelectronic states. ${ }^{34,47}$

\section{Conclusion}

In summary, quantum confinements and dielectric confinements have been investigated for lead halide perovskites with
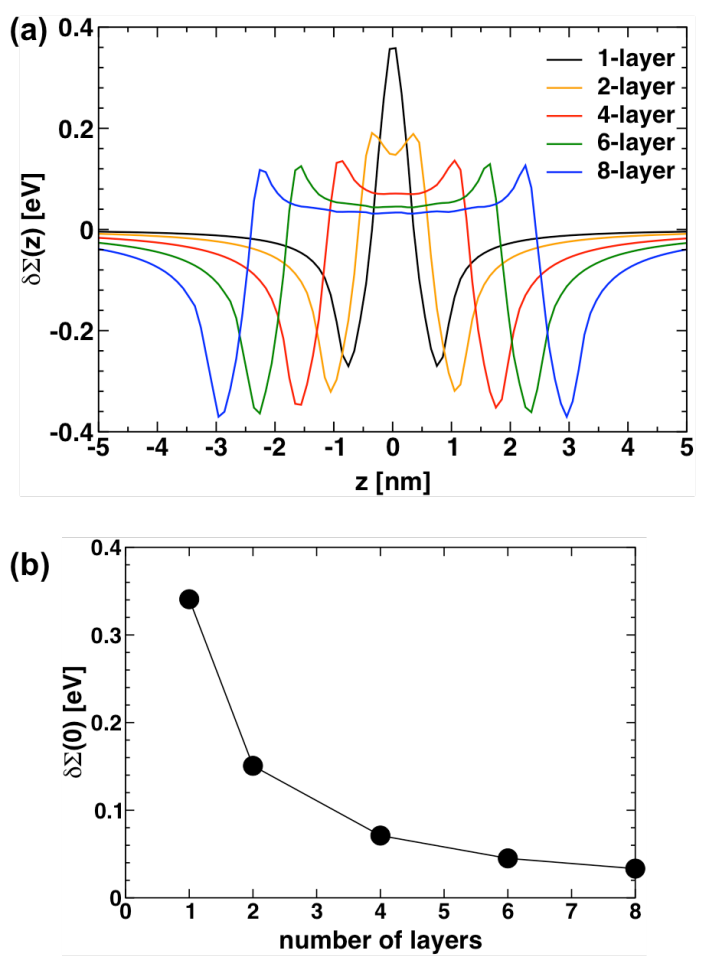

Fig. 9 (a) Self-energy profile $\delta \Sigma(z)$ for slabs of $\mathrm{CH}_{3} \mathrm{NH}_{3} \mathrm{PbI}_{3}$. (b) Self-energy taken at the slab centre $\delta \Sigma(0)$.

inorganic $\left(\mathrm{Cs}^{+}\right)$and organic $\left(\mathrm{CH}_{3} \mathrm{NH}_{3}^{+}\right)$cations for various halogens. An increase of the band gap and a decrease of the dielectric constant occurs upon reducing the thickness of the nanoplatelets. Both quantum and dielectric confinements are predicted to show up for slabs counting less than 4 octahedra in the stacking direction. Dielectric properties in 2D and 2D/3D materials are discussed as well. In all cases, the dramatic effect of phonons on the dielectric constant has been illustrated with a great enhancement for the inorganic parts of the materials. This study is a first step towards the computation of self-energy corrections ${ }^{34}$ that are necessary to reach an accurate description of the exciton in these hybrid materials. Devices involving HOP imply an interface with other semiconductors, e.g. $\mathrm{TiO}_{2} \cdot{ }^{80,81}$ As with 2D HOP materials, a dielectric contrast can then have dramatic effects. These interfaces will be the focus of upcoming studies.

\section{Acknowledgement}

This work has been supported by University of Rennes 1 (Action Incitative, Défis Scientifiques Emergents 2015). It was performed, in part, at the Center for Integrated Nanotechnologies, an Office of Science User Facility operated for the U.S. Department of Energy (DOE) Office of Sci- 
ence by Los Alamos National Laboratory (Contract DEAC52-06NA25396). This work was granted access to the HPC resources of [TGCC/CINES/IDRIS] under the allocation 2015096724 by GENCI. J.E. and L.P. acknowledge the support from SNAP French ANR Projects. J. E. work is also supported by the Fondation d'entreprises banque Populaire de l'Ouest under Grant PEROPHOT 2015.

\section{References}

1 A. Kojima, K. Teshima, Y. Shirai and T. Miyasaka, J. Am. Chem. Soc., 2009, 131, 6050 .

2 H. Zhou, Q. Chen, G. Li, S. Luo, T. Song, H.-S. Duan, Z. Hong, J. You, Y. Liu and Y. Yang, Science, 2014, 345, 542.

3 M. R. Filip, G. E. Eperon, H. J. Snaith and F. Giustino, Nat. Commun., 2014, 5, 5757.

4 C. Katan, L. Pedesseau, M. Kepenekian, A. Rolland and J. Even, J. Mater. Chem. A, 2015, 3, 9232.

5 D. B. Mitzi, C. A. Feild, W. T. A. Harrison and A. M. Guloy, Nature, 1994 369, 467.

6 C. R. Kagan, D. B. Mitzi and C. D. Dimitrakopoulos, Science, 1999, 286 945

7 K. Chondroudis and D. B. Mitzi, Chem. Mater., 1999, 11, 3028.

8 J. Wenus, R. Parashkov, S. Ceccarelli, A. Brehier, J.-S. Lauret, M. S. Skolnick, E. Deleporte and D. G. Lidzey, Phys. Rev. B, 2006, 74, 235212

9 I. Koutselas, P. Bampoulis, E. Maratou, T. Evagelinou, G. Pagona and G. C. Papavassiliou, J. Phys. Chem. C, 2011, 115, 8475.

10 G. C. Papavassiliou, G. Pagona, N. Karousis, G. A. Mousdis, I. Koutselas and A. Vassilakopoulou, J. Mater. Chem., 2012, 22, 8271.

11 J. Even, L. Pedesseau, C. Katan, M. Kepenekian, J.-S. Lauret, D. Sapori and E. Deleporte, J. Phys. Chem. C, 2015, 119, 10161.

12 L. C. Schmidt, A. Pertegás, S. González-Carrero, O. Malinkiewicz, S. Agouram, G. Mínguez Espallargas, H. J. Bolink, R. E. Galian and J. Pérez-Prieto, J. Am. Chem. Soc., 2014, 136, 850.

13 F. Zhang, H. Zhong, C. Chen, X. Wu, X. Hu, H. Huang, J. Han, B. Zou and Y. Dong, ACS Nano, 2015, 4, 4533.

14 Y. Fu, F. Meng, M. B. Rowley, B. J. Thompson, M. J. Shearer, D. Ma, R. J. Hamers, J. C. Wright and S. Jin, J. Am. Chem. Soc., 2015, 137, 5810.

15 F. Zhu, L. Men, Y. Guo, Q. Zhu, U. Bhattacharjee, P. M. Goodwin, J. W. Petrich, E. A. Smith and J. Vela, ACS Nano, 2015, 9, 2948.

16 P. Tyagi, S. M. Arveson and W. A. Tisdale, J. Phys. Chem. Lett., 2015, 6 1911.

17 L. Protesescu, S. Yakunin, M. I. Bodnarchuk, F. Krieg, R. Caputo, C. H. Hendon, R. X. Yang, A. Walsh and M. V. Kovalenko, Nano Lett., 2015, 15, 3692 .

18 S. González-Carrero, R. E. Galian and J. Pérez-Prieto, Part. Part. Syst. Charact., 2015, 32, 709.

19 S. Gonzalez-Carrero, R. E. Galian and J. Pérez-Prieto, J. Mater. Chem. A, 2015, 3, 9187.
20 J. A. Sichert, Y. Tong, N. Mutz, M. Vollmer, S. Fischer, K. Z. Milowska, R. Garciá Cortadella, B. Nickel, C. Cardenas-Daw, J. K. Stolarczyk, A. S. Urban and J. Feldmann, Nano Lett., 2015, DOI: 10.1021/acs.nanolett.5b02985.

21 K. Tanaka, T. Takahashi, T. Kondo, T. Umebayashi, K. Asai and K. Ema, Phys. Rev. B, 2005, 71, 045312 .

22 L. Pedesseau, J.-M. Jancu, A. Rolland, E. Deleporte, C. Katan and J. Even, Opt. Quantum Electron., 2014, 46, 1225.

23 D. B. Mitzi, K. Chondroudis and C. R. Kagan, IBM J. Res. Dev., 2001, 45, 29.

24 J. Even, L. Pedesseau and C. Katan, ChemPhysChem, 2014, 15, 3733.

25 J. Even, L. Pedesseau, M.-A. Dupertuis, J.-M. Jancu and C. Katan, Phys. Rev. B, 2012, 86, 205301.

26 F. J. A. Solsona and J. M. Fornies-Marquina, J. Phys. Appl. Phys., 1982, 15, 1783 .

27 C. C. Desai, J. L. Rai and A. D. Vyas, J. Mater. Sci., 1982, 17, 3249.

28 J. Even, L. Pedesseau and C. Katan, J. Phys. Chem. C, 2014, 118, 11566.

29 A. Miyata, A. Mitioglu, P. Plochocka, O. Portugall, J. T.-W. Wang, S. D. Stranks, H. J. Snaith and R. J. Nicholas, Nature Phys., 2015, 11, 582.

30 H.-H. Fang, R. Raissa, M. Abdu-Aguye, S. Adjokatse, G. R. Blake, J. Even and M. A. Loi, Adv. Funct. Mater., 2015, 25, 2378-2385.

31 D. A. Valverde-Chávez, C. Ponseca Jr., C. Stoumpos, A. Yartsev, M. G. Kanatzidis, V. Sundström and D. G. Cooke, arXiv:1507.02179 [condmat.mtrl-sci].

32 B. A. Gregg, J. Phys. Chem. B, 2003, 107, 4688.

33 E. A. Muljarov, S. G. Tikhodeev and N. A. Gippius, Phys. Rev. B, 1995, 51, 14370.

34 J. Even, L. Pedesseau and M. Kepenekian, Phys. Chem. Chem. Phys., 2014, 16, 25182.

35 J. M. Soler, E. Artacho, J. D. Gale, A. García, J. Junquera, P. Ordejón and D. Sánchez-Portal, J. Phys.: Condens. Matter, 2002, 14, 2745.

36 E. Artacho, E. Anglada, O. Diéguez, J. D. Gale, A. García, J. Junquera, R. M. Martin, P. Ordejón, J. M. Pruneda, D. Sánchez-Portal and J. M. Soler, J. Phys.: Condens. Matter, 2008, 20, 064208.

37 J. P. Perdew, K. Burke and M. Ernzerhof, Phys. Rev. Lett., 1996, 77, 3865.

38 N. Troullier and J. L. Martins, Phys. Rev. B, 1991, 43, 1993.

39 E. Artacho, D. Sánchez-Portal, P. Ordejón, A. García and J. M. Soler, phys. stat. sol. (b), 1999, 215, 809.

40 J. Even, L. Pedesseau, J.-M. Jancu and C. Katan, J. Phys. Chem. Lett., 2013, 4, 2999.

41 J. Even, L. Pedesseau, J.-M. Jancu and C. Katan, Phys. Status Solidi RRL, $2014,8,31$

42 P. Umari, E. Mosconi and F. De Angelis, Sci. Rep., 2014, 4, 4467.

43 A. Amat, E. Mosconi, E. Ronca, C. Quarti, P. Umari, M. K. Nazeeruddin, M. Grätzel and F. De Angelis, Nano Lett., 2014, 14, 3608. 
44 F. Brivio, K. T. Butler, A. Walsh and M. van Schilfgaarde, Phys. Rev. B, 2014, 89, 155204.

45 R. G. Barrera and C. B. Duke, Phys. Rev. B, 1976, 13, 4477.

46 M. Kumagai and T. Takagahara, Phys. Rev. B, 1989, 40, 12359.

47 R. Benchamekh, N. A. Gippius, J. Even, M. O. Nestoklon, J.-M. Jancu, S. Ithurria, B. Dubertret, A. L. Efros and P. Voisin, Phys. Rev. B, 2014, 89, 035307 .

48 B. Meyer and D. Vanderbilt, Phys. Rev. B, 2001, 63, 205426.

49 F. Giustino and A. Pasquarello, Phys. Rev. B, 2005, 71, 144104.

50 B. Lee, C.-K. Lee, S. Han, J. Lee and C. S. Hwang, J. Appl. Phys., 2008, 103, 024106 .

51 G. Kresse and J. Furthmüller, Phys. Rev. B, 1996, 54, 11169.

52 P. Giannozzi, S. Baroni, N. Bonini, M. Calandra, R. Car, C. Cavazzoni, D. Ceresoli, G. L. Chiarotti, M. Cococcioni, I. Dabo, A. Dal Corso, S. de Gironcoli, S. Fabris, G. Fratesi, R. Gebauer, U. Gerstmann, C. Gougoussis, A. Kokalj, M. Lazzeri, L. Martin-Samos, N. Marzari, F. Mauri, R. Mazzarello, S. Paolini, A. Pasquarello, L. Paulatto, C. Sbraccia, S. Scandolo, G. Sclauzero, A. P. Seitsonen, A. Smogunov, P. Umari and R. M. Wentzcovitch, J. Phys.: Condens. Matter, 2009, 21, 395502.

53 N. Shi and R. Ramprasad, Appl. Phys. Lett., 2005, 87, 262102.

54 N. Shi and R. Ramprasad, Phys. Rev. B, 2006, 74, 045318.

55 F. Giustino, P. Umari and A. Pasquarello, Phys. Rev. Lett., 2003, 91, 267601 .

56 J. Junquera, M. H. Cohen and K. M. Rabe, J. Phys.: Condens. Matter, 2007, 19, 213203.

57 A. Ron, Phys. Rev., 1963, 131, 2041-2045.

58 E. K. U. Gross, J. F. Dobson and M. Petersilka, Top. Curr. Chem., 1996, 181, 81 .

59 F. Calvayrac, P.-G. Reinhard, E. Suraud and C. A. Ullrich, Phys. Rep., 2000, 337, 493-578.

60 T. Otobe, M. Yamagiwa, J.-I. Iwata, K. Yabana, T. Nakatsukasa and G. F. Bertsch, Phys. Rev. B, 2008, 77, 165104.

61 D. N. Basov, R. D. Averitt, D. van der Marel, M. Dressel and K. Haule, Rev. Mod. Phys., 2011, 83, 471-541.
62 Y.-S. Park, S. Guo, N. S. Makarov and V. I. Klimov, ACS Nano, 2015, DOI: 10.1021/acsnano.5b04584.

63 D. M. Trots and S. V. Myagkota, J. Phys. Chem. Solids, 2008, 69, 2520.

64 T. Baikie, Y. Fang, J. M. Kadro, M. Schreyer, F. Wei, S. G. Mhaisalkar, M. Grätzel and T. J. White, J. Mater. Chem. A, 2013, 1, 5628-5641.

65 G. C. Papavassiliou, G. A. Mousdis, C. P. Raptopoulou and A. Terzis, Z. Naturforsch., 1999, 54, 1405-1409.

66 A. Lemmerer and D. G. Billing, Dalton Trans., 2012, 41, 1146-1157.

67 D. G. Billing and A. Lemmerer, Acta Crystallogr. B, 2007, 63, 735-747.

68 G. C. Papavassiliou, G. A. Mousdis, C. P. Raptopoulou and A. Terzis, Z. Naturforsch., 2000, 55, 536-540.

69 I. P. Swainson, R. P. Hammond, C. Soullière, O. Knop and W. Massa, J. Solid State Chem., 2003, 176, 97.

70 Y. H. Chang, C. H. Park and K. Matsuishi, J. Korean Phys. Soc., 2004, 44, 889-893.

71 R. A. Jishi, O. B. Ta and A. A. Sharif, J. Phys. Chem. C, 2014, 118, 28344 28349.

72 C. Grote and R. F. Berger, J. Phys. Chem. C, 2015, 119, 22832-22837.

73 W.-J. Yin, T. Shi and Y. Yan, J. Phys. Chem. C, 2015, 119, 5253.

74 K. Wakamura and Y. Noda, J. Phys. Chem. Solids, 2001, 62, 2027.

75 M. Hirasawa, T. Ishihara, T. Goto, K. Uchida and N. Miura, Phys. B, 1994, 201, 427.

76 F. Brivio, A. B. Walker and A. Walsh, APL Mater., 2013, 1, 042111.

77 A. Poglitsch and D. Weber, J. Chem. Phys., 1987, 87, 6373.

78 N. Onoda-Yamamuro, T. Matsuo and H. Suga, J. Phys. Chem. Solids, 1992, 53, 935 .

79 Q. Lin, A. Armin, R. C. R. Nagiri, P. L. Burn and P. Meredith, Nat. Photonics, 2014, 9, 106 .

80 V. Roiati, E. Mosconi, A. Listorti, S. Colella, G. Gigli and F. De Angelis, Nano Lett., 2014, 14, 2168.

81 E. Mosconi, E. Ronca and F. De Angelis, J. Phys. Chem. Lett., 2014, 5, 2619. 